\title{
Influence of Hydroxypropyl Methylcellulose on Metronidazole Crystallinity in Spray-Congealed Polyethylene Glycol Microparticles and Its Impact with Various Additives on Metronidazole Release
}

\author{
Ching Mien Oh, ${ }^{1}$ Paul Wan Sia Heng, ${ }^{1}$ and Lai Wah Chan ${ }^{1,2}$
}

Received 7 January 2015; accepted 6 April 2015; published online 2 May 2015

\begin{abstract}
The purpose of this study was to investigate the effect of a hydrophilic polymer, hydroxypropyl methylcellulose (HPMC), on the crystallinity and drug release of metronidazole (MNZ) in spraycongealed polyethylene glycol (PEG) microparticles and to further modify the drug release using other additives in the formulation. HPMC has been used in many pharmaceutical formulations and processes but to date, it has not been employed as an additive in spray congealing. Crystallinity of a drug is especially important to the development of pharmaceutical products as active pharmaceutical ingredients (APIs) are mostly crystalline in nature. A combination of X-ray diffractometry, differential scanning calorimetry, Raman spectroscopy and Fourier transform-infrared spectroscopy (FT-IR) spectroscopy was employed to investigate the degree of crystallinity and possible solid-state structure of MNZ in the microparticles. The microparticles with HPMC were generally spherical. Spray congealing decreased MNZ crystallinity, and the presence of HPMC reduced the drug crystallinity further. The reduction in MNZ crystallinity was dependent on the concentration of HPMC. Smaller HPMC particles also resulted in a greater percentage reduction in MNZ crystallinity. Appreciable modification to MNZ release could be obtained with HPMC. However, this was largely attributed to the role of HPMC in forming a diffusion barrier. Further modification of drug release from spray-congealed PEG-HPMC microparticles was achieved with the addition of $5 \% \mathrm{w} / \mathrm{w}$ dicalcium phosphate but not with magnesium stearate, methyl cellulose, polyvinylpyrrolidone, silicon dioxide and sodium oleate/citric acid. Dicalcium phosphate facilitated formation of the diffusion barrier.
\end{abstract}

KEY WORDS: crystallinity; drug release; hydroxypropyl methylcellulose; metronidazole; spray congealing.

\section{INTRODUCTION}

The form of drug, amorphous or crystalline, is especially important to the development of pharmaceutical products. Varying degrees of drug amorphism can be generated through pharmaceutical processes such as milling, wet granulation, drying and compaction (1). The amorphous content of the drug can affect the properties of the resultant product. For example, it can confer advantages such as improvement in dissolution where poor water solubility is a concern and enhancement of bioavailability where drug absorption is the rate limiting step $(2,3)$. Many modern drug candidates are hydrophobic in nature and therefore, utilising the amorphous form of drugs is a powerful approach in addressing

\footnotetext{
${ }^{1}$ GEA-NUS Pharmaceutical Processing Research Laboratory, Department of Pharmacy, Faculty of Science, National University of Singapore, 18 Science Drive 4, Singapore, 117543, Singapore.

${ }^{2}$ To whom correspondence should be addressed. (e-mail: phaclw@nus.edu.sg)
}

the poor administration, distribution, metabolism and elimination (ADME) profiles of these drugs.

While amorphism of drugs can be induced during product development, it is also important to inhibit crystallisation of the amorphous drug upon dissolution. The higher dissolution rate and apparent solubility of the amorphous drug may cause supersaturation during in vivo dissolution, leading to recrystallisation/precipitation in the gastrointestinal tract and hence, compromise the bioavailability of the drug (1). This is especially evident for BCS class IV substances. Any improvement in dissolution can be masked as the low permeation through the gut wall allows time for recrystallisation of the drug to occur (4).

To understand the underlying mechanism of crystallisation inhibition, the process of nucleation and crystal growth will be discussed. Nucleation occurs when the activation energy of nucleation in a supersaturated solution is surmounted (5). Surfaces and particles will decrease the activation energy and therefore facilitate the nucleation process. The nucleation rate increases with increasing degree of supersaturation and with a decrease in the interfacial energy between the nucleus and solvent. The former factor can be influenced by formulation compounds while the latter factor by the presence of surfactants. Crystal 
growth consists of two steps: (1) diffusion of molecules from the bulk of the solution towards the crystal interface and (2) integration of the molecules into the crystal lattice. The nucleation process and crystal growth can be delayed by drug-polymer interactions, by altering the viscosity of the solution and by adsorption of the polymer on the nucleus or the growing crystal, thereby inhibiting drug crystallisation $(1,6-8)$.

One study has shown that hydroxypropyl methylcellulose (HPMC) is able to induce the amorphism of drugs (9). It was found that HPMC could inhibit the crystallisation of amorphous drugs, such as albendazole, by reducing the nucleation rate (10). It also had the ability to stabilise the amorphous drugs. It was speculated that HPMC affected the nucleation kinetics by increasing the kinetic barrier to nucleation. The effect was proportional to the concentration of HPMC (11).

Many researchers have studied the use of HPMC as a coating polymer in various processes, but little consideration has been given to the use of HPMC as an additive in spray congealing. Spray congealing is a process whereby a molten mixture, at temperature above its melting point, is atomized into fine droplets in a cooling chamber. Upon contact with cool air, the droplets quickly solidify to form microparticles. One of the advantages of spray congealing includes the production of microparticles without the use of water or organic solvents (12), making spray congealing an environmentally friendly process that is attractive for the processing of moisture-sensitive drugs. It also eliminates the drying step and the risk associated with any residual organic solvent that comes with the solvent evaporation method (13). Conventionally, the molten mixture consists of a drug melted, dissolved or dispersed in a carrier material. Carrier materials can be hydrophilic such as polyethylene glycol (PEG) and stearoyl polyoxylglycerides or lipophilic such as stearic acid, hydrogenated soybean oil and carnauba wax. Savolainen et al. have found that the drug crystallinity of spray-congealed products plays a more significant role in drug release than the matrix lipophilicity (14). However, some studies have reported that the spray congealing process does not affect the crystallinity of the drug $(15,16)$.

To date, no studies have investigated the effects of HPMC as an additive in spray-congealed microparticles. The use of HPMC in combination with various additives in the modification of the drug release from the microparticles has not been explored. Therefore, in this study, the influence of HPMC on the crystallinity and drug release of metronidazole in spray-congealed microparticles was investigated. The impact of different particle size of HPMC on drug crystallinity and their mechanism of inhibiting drug recrystallisation was explored. The influence of various additives and their mechanism of action on the drug release from these microparticles were also investigated.

\section{MATERIALS AND METHODS}

\section{Materials}

Metronidazole (MNZ), a yellowish crystalline powder (Sunward Pharmaceutical, Singapore), was used as a model drug in this study. Polyethylene glycol 3350 (PEG 3350; m.p. $53-59^{\circ} \mathrm{C}$ ) was supplied by Clariant GMBH (Germany) and hydroxypropyl methylcellulose K15M by Dow Chemical (USA). Other additives used were polyvinylpyrrolidone K90 (PVP-K90; ISP Technologies, USA), methyl cellulose 13-18 cps (MC; Tokyo Chemical Industry, Japan), silicon dioxide $\left(\mathrm{SiO}_{2}\right.$; Fuji Chemical Industry, Japan), magnesium stearate (MgSt; Productos Metalest, Spain), dicalcium phosphate (DCP; Rhodia, USA), citric acid (Merck, Germany) and sodium oleate (BDH Chemicals, England). Hydrochloric acid $(\mathrm{HCl})$, potassium dihydrogen phosphate, sodium hydroxide (Merck, Germany) and deionised water (Millipore, France) were used to prepare the test media.

\section{Preparation of Spray-Congealed Microparticles}

Microparticles composed of PEG and HPMC were prepared by spray congealing (Mobile MinorTM 2000, GEA Niro, Soeborg, Denmark). PEG was weighed in a glass beaker and melted in the oven (UL 40, Memmert, Schwabach, Germany) before transferring to an oil bath kept at $80^{\circ} \mathrm{C}$. The required amount of MNZ and/or HPMC was then added to the molten PEG and mixed using a magnetic stirrer. When required, the various additives were added last to the molten mixture. The molten mixture was fed through a peristaltic pump at a feed rate of $20 \mathrm{~mL} / \mathrm{min}$ and atomized counter-current as a fountain of droplets in the cooling chamber of the spray congealer via a two-fluid nozzle. The spray delivery system temperature was set at $80^{\circ} \mathrm{C}$ to avoid the solidification of the molten mixture, with atomisation pressure set at 2.5 bar. Upon contact with cool air $\left(5^{\circ} \mathrm{C}\right)$, the droplets solidified, and the microparticles formed were collected by a base container while fines were collected by a cyclone. The composition of different formulations is shown in Table I.

\section{Particle Size Analysis}

Measurements of HPMC particle size were carried out using laser diffractometry (LS230, Coulter, USA) with a dry powder module. Size determinations were carried out in triplicates and the results averaged. The median particle size, $d_{50}$, was derived from the size distribution plots.

\section{Morphology of Microparticles}

The morphology of spray-congealed microparticles was visualised using a scanning electron microscope (JSM-6010LV, JEOL, Japan). The microparticles were mounted on aluminium studs using conductive carbon tapes and observed at $1.5 \mathrm{kV}$ under vacuum.

\section{Thermal Analysis}

The thermal characteristics of the single components, physical mixtures and spray-congealed microparticles were determined using a differential scanning calorimeter (DSC60, Shimadzu, Japan). A sealed aluminium pan loaded with sample was placed in a DSC furnace and heated from 20 to $200^{\circ} \mathrm{C}$ at a heating rate of $5^{\circ} \mathrm{C} / \mathrm{min}$. An empty sealed aluminium pan was used as a reference. Measurements were carried out in triplicates and the results averaged. 
Table I. Composition of Different Spray-Congealed Microparticles with Drug

\begin{tabular}{llllcl}
\hline Formulation code & PEG $(\%, w / w)$ & MNZ $(\%, w / w)$ & HPMC $(\%, w / w)$ & Drug content $(\%, w / w)$ & Encapsulation efficiency $(\%)$ \\
\hline P & 100 & 0 & 0 & - & - \\
PM & 90 & 10 & 0 & $9.19 \pm 0.10$ & $91.92 \pm 0.97$ \\
$\mathrm{PMH}_{5}$ & 85 & 10 & 5 & $8.28 \pm 0.17$ & $82.78 \pm 1.73$ \\
$\mathrm{PMH}_{10}$ & 80 & 10 & 10 & $8.62 \pm 0.34$ & $86.18 \pm 3.41$ \\
$\mathrm{PMH}_{15}$ & 75 & 15 & $7.30 \pm 0.27$ & $72.95 \pm 2.73$ \\
\hline
\end{tabular}

$P E G$ polyethylene glycol, $M N Z$ metronidazole, $H P M C$ hydroxypropyl methylcellulose, $P$ polyethylene glycol only, $P M$ polyethylene glycol and metronidazole, $P M H$ polyethylene glycol, metronidazole and hydroxypropyl methylcellulose

\section{X-ray Powder Diffraction Analysis}

The single components, physical mixtures and spraycongealed microparticles were analysed using an X-ray powder diffractometer (XRD-6000, Shimadzu, Japan) with $\mathrm{Cu} \mathrm{K} \alpha$ radiation $(\lambda=1.5406 \AA)$, voltage of $40 \mathrm{kV}$ and current of $30 \mathrm{~mA}$. The scanning angle $(2 \theta)$ ranged from 10 to $35^{\circ}$ with a scanning rate of $2 \% \mathrm{~min}$. Measurements were performed in triplicates and the results averaged. The intensity of the peak obtained at $12.5^{\circ}$ corresponded to the degree of MNZ crystallinity. The percentage reduction in MNZ crystallinity was calculated from the following equation:

Percentage reduction in MNZ crystallinity $=\left(P_{\mathrm{p}}-P_{\mathrm{m}}\right) / P_{\mathrm{p}} \times 100 \%$

where $P_{\mathrm{p}}$ and $P_{\mathrm{m}}$ are the peak intensity of the physical mixture and the corresponding microparticles at $12.5^{\circ}$. Samples were stored at $25^{\circ} \mathrm{C}$ and $30 \%$ relative humidity (RH). XRD spectra of the samples were obtained periodically to monitor for changes in drug crystallinity.

\section{Raman Spectroscopy}

The single components and microparticles were spread on a glass slide and examined using a Raman spectrometer (XploRA, Horiba Scientific, France), equipped with a 532-nm $\mathrm{He} / \mathrm{Ne}$ laser source $(30 \mathrm{~mW}), 12001 / \mathrm{nm}$ grating and a microscope system (BX 41, Olympus, Japan). Each spectrum represented the average of three measurements.

\section{Fourier Transform-Infrared Spectroscopy (FT-IR)}

Interaction among MNZ, HPMC and PEG in the microparticles was investigated using FT-IR spectroscopy (Spectrum 100, Perkin Elmer, USA). Samples of the single components, physical mixtures and microparticles were each mixed with $\mathrm{KBr}$ in the weight ratio of 1:99 and ground in a mortar before being compressed into a tablet, using a manual hydraulic press (Atlas Series, Specac, England) at 10,000 kg for $5 \mathrm{~min}$. Infrared spectra of the samples were obtained and analysed.

\section{Determination of Drug Content and Encapsulation Efficiency}

The drug-loaded microparticles were accurately weighed and diluted with $0.1 \mathrm{M} \mathrm{HCl}$ to a specified volume in a flask. The test mixture was agitated at 60 oscillations $/ \mathrm{min}$ for $30 \mathrm{~min}$ in a shaker bath (M20S, MT/2, Lauda, Germany) at $65^{\circ} \mathrm{C}$. It was then cooled to room temperature before an aliquot sample was passed through a $0.45-\mu \mathrm{m}$ membrane filter (Sartorius, Germany). The liquid sample was then diluted appropriately for drug assay by UV spectrophotometry (UV 1201, Shimadzu, Japan) at $277 \mathrm{~nm}$. Drug content was expressed as the amount of drug encapsulated per unit weight of microparticles. The drug content was determined in triplicates and the results averaged. The encapsulation efficiency (EE) was calculated as follows:

$\mathrm{EE}(\%)=W_{\mathrm{a}} / W_{\mathrm{t}} \times 100$

where $W_{\mathrm{a}}$ is the actual drug content and $W_{\mathrm{t}}$ is the theoretical drug content, obtained from the amount of drug employed in the formulation.

\section{Dissolution of Microparticles}

Drug release studies for pure drug and spray-congealed microparticles were carried out using the United States Pharmacopeia (USP) dissolution test method (apparatus 2) with paddles rotating at $50 \mathrm{rpm}$. The different batches of microparticles were sieved to obtain samples with similar particle size. A dissolution test unit (Caleva 9ST Dissolution Tester, Frankfurt, Germany) with an automated sampler (Caleva, England) was used. The dissolution medium used was $900 \mathrm{ml}$ of phosphate buffer ( $\mathrm{pH} 7.4$ ) at $37^{\circ} \mathrm{C}$. Samples of dissolution medium were withdrawn at specific time intervals from each dissolution vessel and analysed spectrophotometrically (UV 1201, Shimadzu, Japan) at a wavelength of $319 \mathrm{~nm}$. The percentage of drug released with respect to the total drug content was calculated. Dissolution test was carried out in triplicates and the results averaged.

\section{Statistical Analysis}

The results were analysed using one-way analysis of variance (ANOVA) at level of significance 0.05 with the aid of statistical analysis software (SPSS, version 13.0, USA), where appropriate.

\section{RESULTS}

\section{Morphology of Spray-Congealed Microparticles}

Spray-congealed microparticles of all the formulations were examined under the light microscope. Microparticles with and without HPMC were generally spherical and discrete (Fig. 1). The molten mixture consisting of PEG and HPMC 

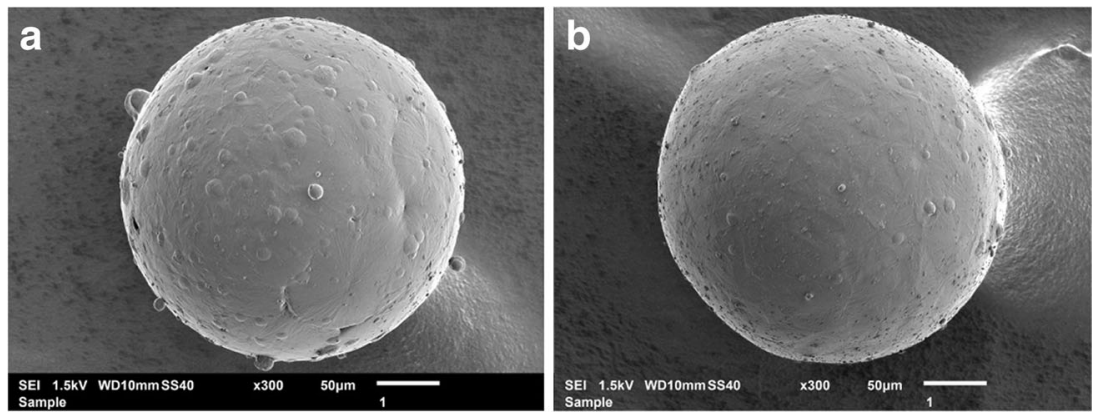

Fig. 1. Photomicrographs of microparticles a without HPMC and b with HPMC

appeared as a white, homogeneous suspension. Upon addition of metronidazole, the mixture turned yellowish. Similarly, microparticles containing MNZ appeared yellowish in colour while those without MNZ were white.

\section{X-ray Powder Diffraction}

X-ray diffraction spectra of single components showed the crystalline nature of PEG and MNZ, with major peaks at 19.3 and $23.4^{\circ}$ for PEG and major peaks at 12.5, 14, 24.8, 29.4 and $33.3^{\circ}$ for MNZ (Fig. 2a). HPMC had no distinct peaks which reflected its amorphous nature. Figure $2 \mathrm{~b}$ shows the XRD spectra of the freshly spray-congealed microparticles and their corresponding physical mixtures.

The different particle size of HPMC might influence the nucleation and crystal growth of MNZ to varying extent in the

\section{a}

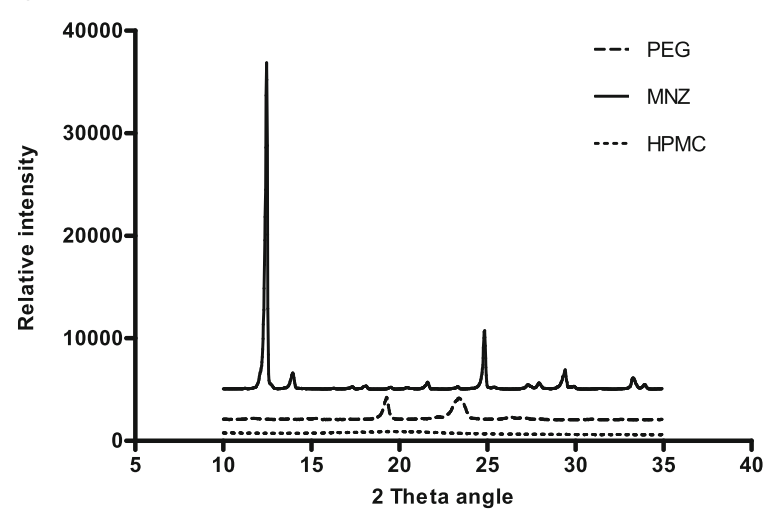

b

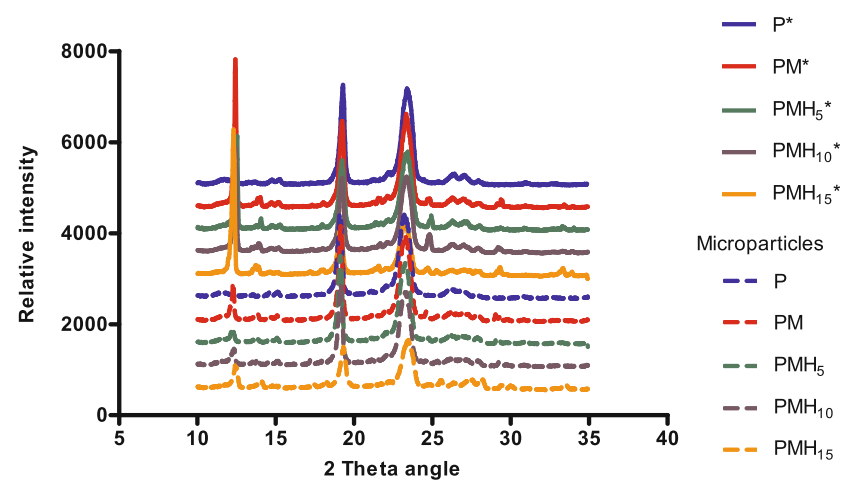

Fig. 2. X-ray diffraction spectra of a single components and $\mathbf{b}$ spraycongealed microparticles and corresponding physical mixtures spray congealing process. To study this effect, it is important to ensure that MNZ was fully soluble in the molten PEG. The solubility ratio of MNZ to PEG was determined to be $1: 8$ at $100^{\circ} \mathrm{C}$. Therefore, the formulations in this study contained $10 \% \mathrm{MNZ}$ and at least $80 \%$ PEG. HPMC K15M of various mesh sizes was added at 5 or $10 \%$ to produce spray-congealed microparticles (Table II).

The microparticles were stored at $25^{\circ} \mathrm{C}$ and $30 \% \mathrm{RH}$ and their MNZ crystallinity monitored over a period of 3 months. MNZ crystallinity was indicated by the peak intensity at $12.5^{\circ}$ (Fig. 3). No particular trend was observed in the peak intensities of the formulations at the various time intervals. Further analysis was carried out by calculating the percentage reduction in MNZ crystallinity of the microparticles with respect to the corresponding physical mixture. To enable more meaningful data visualisation, a scatter plot of the data points was constructed (Fig. 4).

\section{Differential Scanning Calorimetry}

Figure 5a shows the DSC curves of the single components. PEG and MNZ showed a strong endothermic peak at about $60^{\circ} \mathrm{C}$ and about $165^{\circ} \mathrm{C}$, respectively. HPMC showed a broad peak which reflected its amorphous nature. The physical mixtures and microparticles consisted of $10 \%$ MNZ. However, the physical mixtures of polyethylene glycol and metronidazole $\left(\mathrm{PM}^{*}\right)$ and polyethylene glycol, metronidazole and hydroxypropyl methylcellulose $\left(\mathrm{PMH}_{5} *\right)$ did not show any noticeable MNZ endothermic peak upon melting (Fig. 5b). This could be attributed to the drug dissolving into PEG when the sample was heated.

Table II. Formulations Containing HPMC K15M of Different Mesh Sizes and Median Particle Size

Median HPMC

Formulation $\quad$ HPMC mesh size $(\mu \mathrm{m})$ particle size $\pm \mathrm{SD}(\mu \mathrm{m})^{a}$

\begin{tabular}{lcr}
\hline Control (no HPMC) & - & - \\
A & $20-30$ & $33 \pm 0.3$ \\
B & $40-50$ & $59 \pm 0.2$ \\
C & $70-80$ & $114 \pm 1.6$ \\
D & $100-120$ & $181 \pm 4.3$ \\
E & $160-200$ & $275 \pm 3.7$ \\
\hline
\end{tabular}

HPMC hydroxypropyl methylcellulose

${ }^{a}$ Median particle size determined experimentally by laser diffractometry 


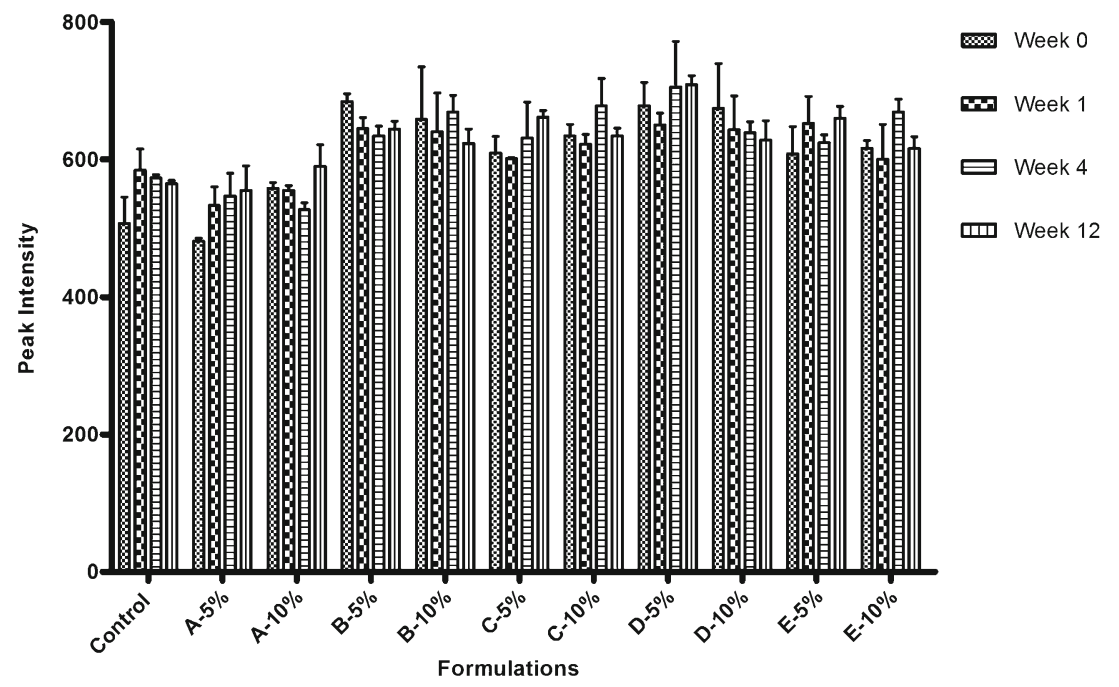

Fig. 3. Peak intensities in XRD spectra of various formulations monitored over a period of 3 months at $25^{\circ} \mathrm{C}$ and $30 \% \mathrm{RH}$

\section{Raman and FT-IR Spectroscopies}

The Raman spectra of the single components are shown in Fig. 6a-c, while those of $\mathrm{PM}, \mathrm{PMH}_{5}, \mathrm{PMH}_{10}$ and $\mathrm{PMH}_{15}$ microparticles are shown in Fig. 6d. Characteristic bands of MNZ, PEG and HPMC are listed in Table III. Raman spectra of MNZ (Fig. 6b) and HPMC (Fig. 6c) exhibit a broad peak at 3441 and $3220 \mathrm{~cm}^{-1}$, respectively, indicative of intermolecular hydrogen bonding of the $\mathrm{OH}$ group. The spectra of $\mathrm{PM}, \mathrm{PMH}_{5}, \mathrm{PMH}_{10}$ and $\mathrm{PMH}_{15}$ (Fig. 6d) showed a shift of the $\mathrm{NO}_{2}(\mathrm{~N}-\mathrm{O}$ stretching) and $\mathrm{OH}$ stretching to a lower wavenumber, indicating the presence of hydrogen bonding among MNZ, PEG and HPMC. The shift in the $\mathrm{OH}$ stretching was greater for $\mathrm{PMH}_{5}, \mathrm{PMH}_{10}$ and $\mathrm{PMH}_{15}$ compared to $\mathrm{PM}$, indicating a larger extent of hydrogen bonding when HPMC was added. FT-IR spectra of MNZ, PEG and HPMC are shown in Fig. 7a-c. Figure $7 d-e$ demonstrates a slight broadening of the $\mathrm{OH}$ stretching of MNZ in the microparticles compared to the



Fig. 4. Scatter plot of drug crystallinity of various formulations. (Asterisk) Points indicate measurements at different time intervals: week 0 (black), week 1 (red), week 4 (green) and week 12 (blue). Horizontal bar indicates mean percentage reduction in drug crystallinity physical mixtures, indicative of the formation of hydrogen bonds among MNZ, PEG and HPMC.

\section{Drug Content and Encapsulation Efficiency}

All the formulations contained 10\% MNZ, but the drug contents of the various microparticles produced were not similar. The drug content and encapsulation efficiency of the microparticles are summarised in Table I. Formulation PM without HPMC had the highest content of MNZ, followed by $\mathrm{PMH}_{10}, \mathrm{PMH}_{5}$ and $\mathrm{PMH}_{15}$. Encapsulation efficiency of
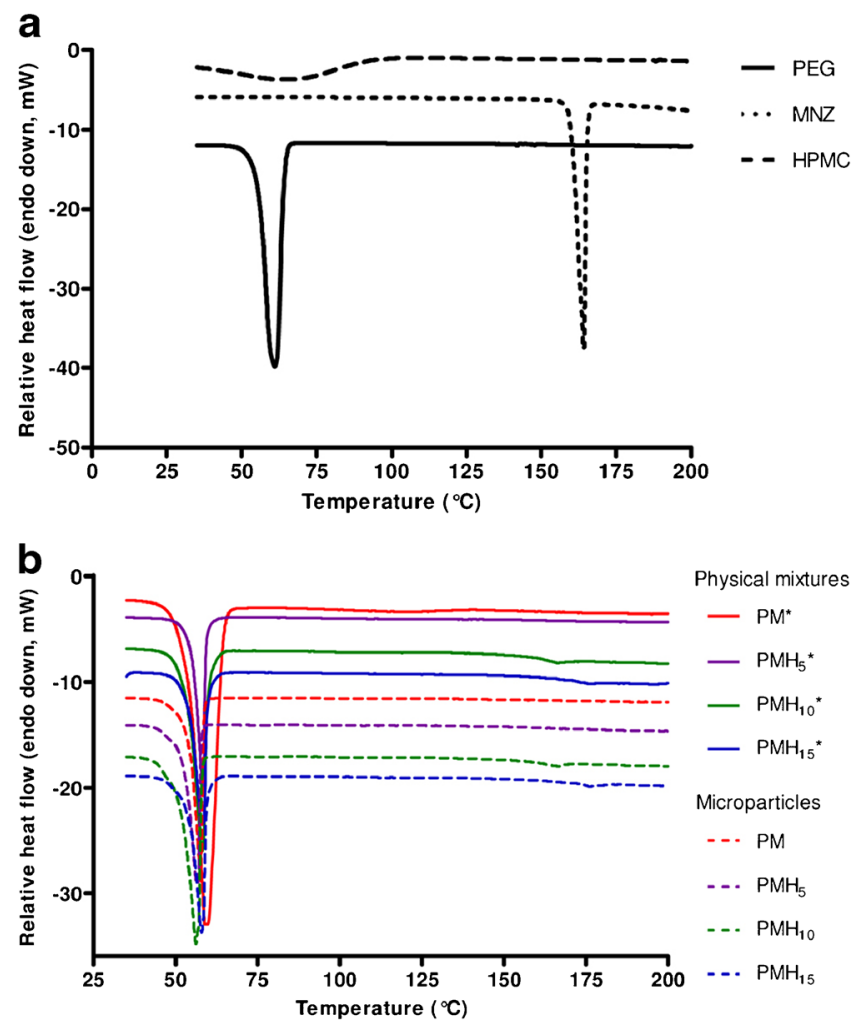

Fig. 5. DSC curves of the a single components and $\mathbf{b}$ spray-congealed microparticles and corresponding physical mixtures 



Fig. 6. Raman spectra of a PEG, b MNZ, c HPMC and d PM, $\mathrm{PMH}_{5}, \mathrm{PMH}_{10}$ and $\mathrm{PMH}_{15}$ microparticles. Note: The Raman spectrum of PM, $\mathrm{PMH}_{5}, \mathrm{PMH}_{10}$ and $\mathrm{PMH}_{15}$ were similar, so only one was shown to avoid duplication

all formulations ranged from 73.0 to $91.9 \%$. Formulations containing $\mathrm{HPMC}\left(\mathrm{PMH}_{5}, \mathrm{PMH}_{10}\right.$ and $\left.\mathrm{PMH}_{15}\right)$ demonstrated a good, albeit lower, encapsulation efficiency of the drug. The drug concentration was kept constant at $10 \%$. Without the addition of HPMC in the formulation, a larger proportion of PEG was available to encapsulate which aptly accounted for the higher encapsulation efficiency. Addition of HPMC to the formulation reduced the proportion of PEG and thus reduced the encapsulation efficiency. When a high concentration of $15 \%$ w/w HPMC was used $\left(\mathrm{PMH}_{15}\right)$, more HPMC and less MNZ particles were encapsulated, resulting in reduced encapsulation efficiency.

Table III. Characteristic Bands of Single Components in Raman and FT-IR Spectra

\section{Single}

component Characteristic bands

Reference

\begin{tabular}{ll}
\hline PEG & $\mathrm{OH}$ group stretching at 3600 to $3200 \mathrm{~cm}^{-1}$ \\
& $\mathrm{R}-\mathrm{CH}_{2}$ stretching at 3000 to $2850 \mathrm{~cm}^{-1}$ \\
& $\mathrm{CH} \mathrm{H}_{2}$ twisting at $1242 \mathrm{~cm}^{-1}$ \\
& $\mathrm{C}-\mathrm{O}$ stretching and $\mathrm{CH}_{2}$ rocking at $1149 \mathrm{~cm}^{-1}$ \\
& $\mathrm{C}-\mathrm{O}-\mathrm{C}$ stretching at $1116 \mathrm{~cm}^{-1}$ \\
& $\mathrm{C}-\mathrm{OH}$ stretching at $1060 \mathrm{~cm}^{-1}$ \\
& $\mathrm{CH}_{2} \mathrm{CH}{ }_{2} \mathrm{OH}$ end group vibration at \\
& approximately $842 \mathrm{~cm}^{-1}$ \\
MNZ & $\mathrm{OH}$ stretching at $3220 \mathrm{~cm}^{-1}$ \\
& $\mathrm{C}=\mathrm{CH}$ stretching at $3101 \mathrm{~cm}^{-1}$ \\
& $\mathrm{NO} 2$ (N-O stretching) at $1528 \mathrm{~cm}^{-1}$ \\
& $\mathrm{C}-\mathrm{OH}$, C-O stretching at $1074 \mathrm{~cm}^{-1}$ \\
& $\mathrm{C}-\mathrm{NO}$ stretching at $864 \mathrm{~cm}^{-1}$ \\
HPMC & $\mathrm{OH}$ stretching at $3441 \mathrm{~cm}^{-1}$ \\
& $\mathrm{C}-\mathrm{H}$ stretching at 3000 to $2800 \mathrm{~cm}^{-1}$ \\
& $\mathrm{C}-\mathrm{O}-\mathrm{C}$ stretching at 1300 to $900 \mathrm{~cm}^{-1}$
\end{tabular}

$P E G$ polyethylene glycol, $M N Z$ metronidazole, HPMC hydroxypropyl methylcellulose

\section{Dissolution of Spray-Congealed Microparticles}

The different batches of microparticles and pure MNZ were sieved to obtain samples with similar particle size range (125 to $250 \mu \mathrm{m}$ ) for dissolution studies at $\mathrm{pH}$ 7.4. The dissolution of the pure MNZ was complete at $15 \mathrm{~min}$ (Fig. 8a). The dissolution profiles were plotted as a function of log time to better differentiate the early phase of dissolution (Fig. 8b).

Since $\mathrm{PMH}_{15}$ had the potential to modify the release of MNZ from the microparticles, HPMC concentration of $15 \%$ was selected for further studies with various additives. Table IV lists the various additives employed in the formulation and the reasons for their selection. Two different concentrations of additives, low (1\%) and high (5\%), were added to the formulation $\mathrm{PMH}_{15}$. The concentration used was limited by the viscosity of the formulation. A high viscosity would cause choking of the delivery tube of the spray congealer.

It should be recalled that PEG is hydrophilic, and it is important that the PEG matrix did not disintegrate before HPMC could hydrate to form the barrier. The modulation of drug release can therefore be achieved by (1) decreasing the gelation time of HPMC or (2) by providing sufficient time for HPMC to swell and form a barrier. The former can be achieved by increasing the speed of HPMC hydration by water while the latter by physically retarding the water from over-hydrating the microparticles, thereby preventing disintegration of PEG and providing more time for HPMC particles to swell and form a barrier.

Figures 9 and 10 show the dissolution profiles of formulations containing the various additives at a low and high additive concentration, respectively.

\section{DISCUSSION}

\section{Crystallinity of MNZ in Spray-Congealed Microparticles}

The drug crystallinity and possible solid-state structrure of MNZ present in the microparticles were investigated using 
a

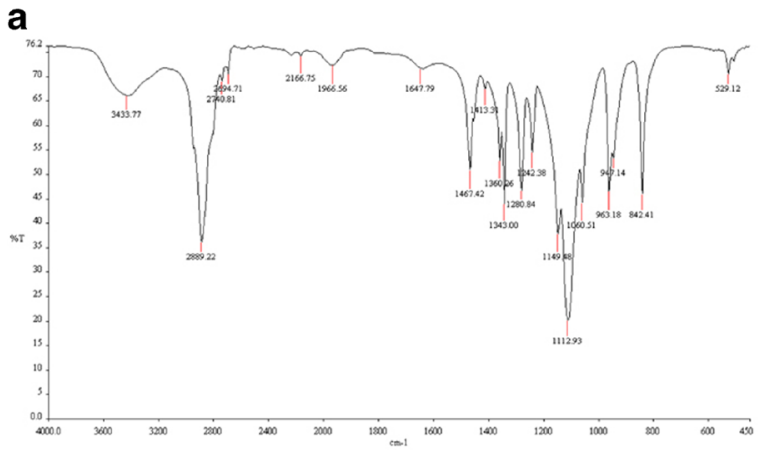

C

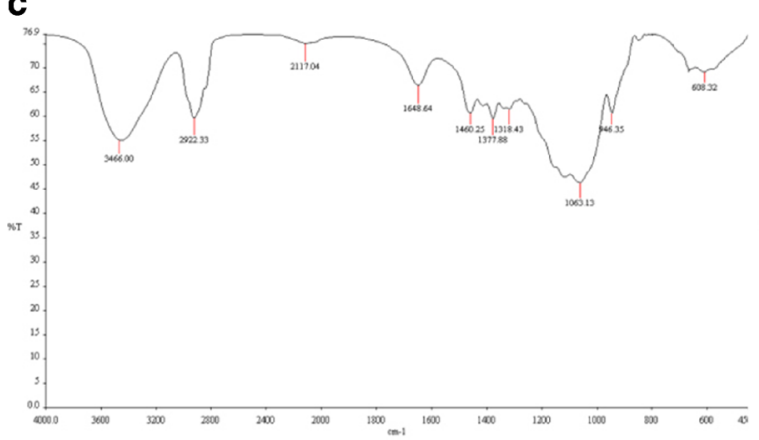

b
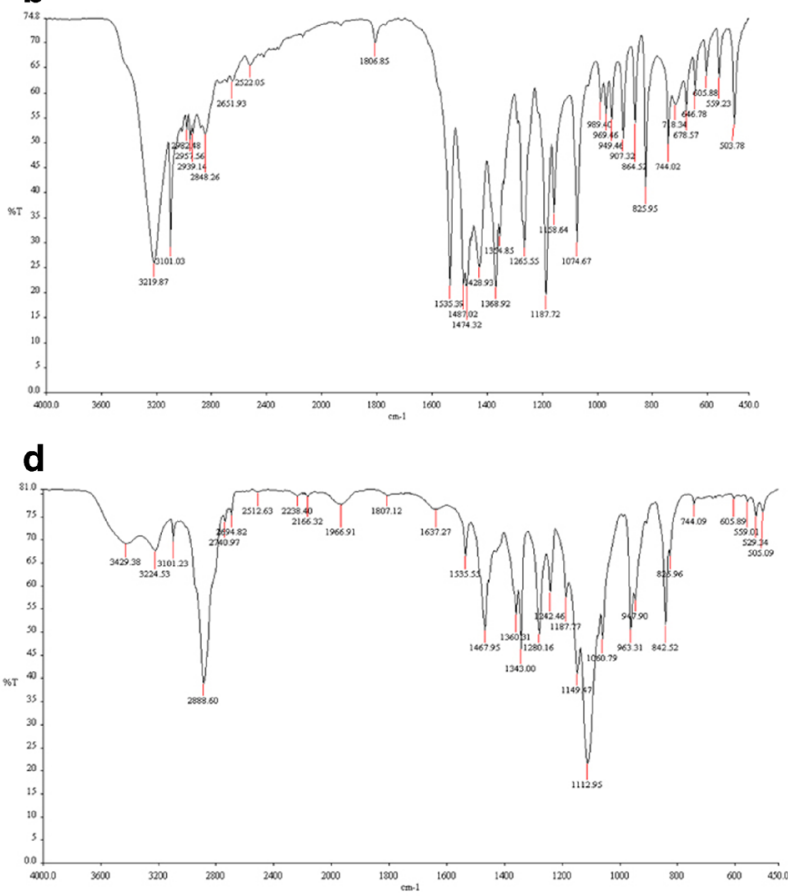

$\mathbf{e}$

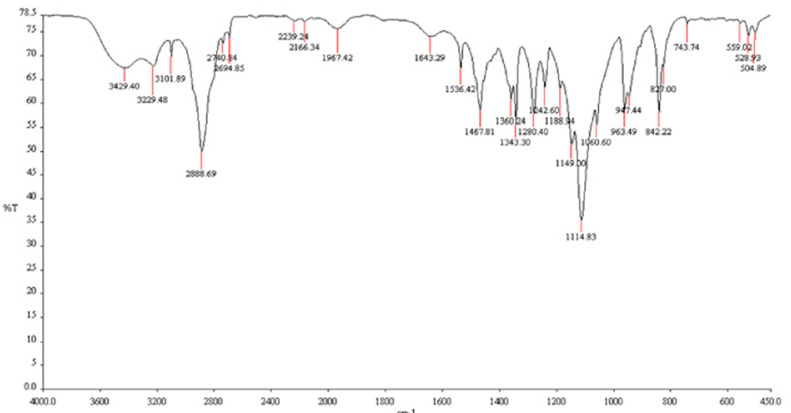

Fig. 7. FT-IR spectra of a PEG, b MNZ, c HPMC, d physical mixtures $\mathrm{PM}^{*}, \mathrm{PMH}_{5} *, \mathrm{PMH}_{10} *$ and $\mathrm{PMH}_{15} *$ and e microparticles $\mathrm{PM}, \mathrm{PMH}_{5}, \mathrm{PMH}_{10}$ and $\mathrm{PMH}_{15}$. Note: All the physical mixtures and microparticles had similar FT-IR spectra. Hence, only one spectrum of the physical mixture and one of the microparticles were shown to avoid duplication

a combination of XRD, DSC, Raman spectroscopy and FT-IR spectroscopy.

The XRD spectrum of P microparticles, comprising solely PEG (Table I), was similar to that of the raw PEG ( $\left.\mathrm{P}^{*}\right)$, indicating that any effect of the spray congealing process on PEG was transitional. Upon melting, PEG molecules were less organised, but upon solidification when the temperature was lowered, the molecules rearranged themselves to form helices that aligned in an orderly fashion, indicating that PEG crystallised easily (21). The PM microparticles consisting of MNZ showed lower MNZ peak intensity in comparison with the corresponding physical mixture (PM*). There were no new peaks observed, indicating the absence of MNZ polymorphic transition. This highlights the ability of the spray congealing process in reducing the drug crystallinity, thereby potentially enhancing dissolution of the drug. The reduction in drug crystallinity was also affirmed in other studies using olanzapine, felodipine, avobenzone and salbutamol sulphate (22-25). However, there were also studies which showed that the spray congealing process had little impact on drug crystallinity of carbamazepine, praziquantel, glimepiride, verapamil or theophylline $(15,16,26-28)$. Various factors can affect the crystallinity of the drug such as the viscosity of the carrier used and the cooling rate of the spray congealing process. Viscosity of a formulation can retard the diffusion of the molecules towards the crystal interface for crystal growth to occur, thereby preventing the rearrangement of drug molecules and resulting in lower drug crystallinity (5). Upon rapid cooling in the spray congealer, the drug molecules, having little time to rearrange themselves, would largely orientate in a haphazard manner. Thus, most of the drug would become amorphous particles or exist as a molecular dispersion, leading to a reduction in the drug crystallinity $(29,30)$. Hence, the crystallinity of a drug may be dependent on the process method besides the formulation employed.

The MNZ peak intensity at $12.5^{\circ}$ for $\mathrm{PMH}_{5}$ (417), $\mathrm{PMH}_{10}$ (458) and $\mathrm{PMH}_{15}$ (594) microparticles was significantly lower $(p<0.05)$ than that of the PM (901) microparticles with 

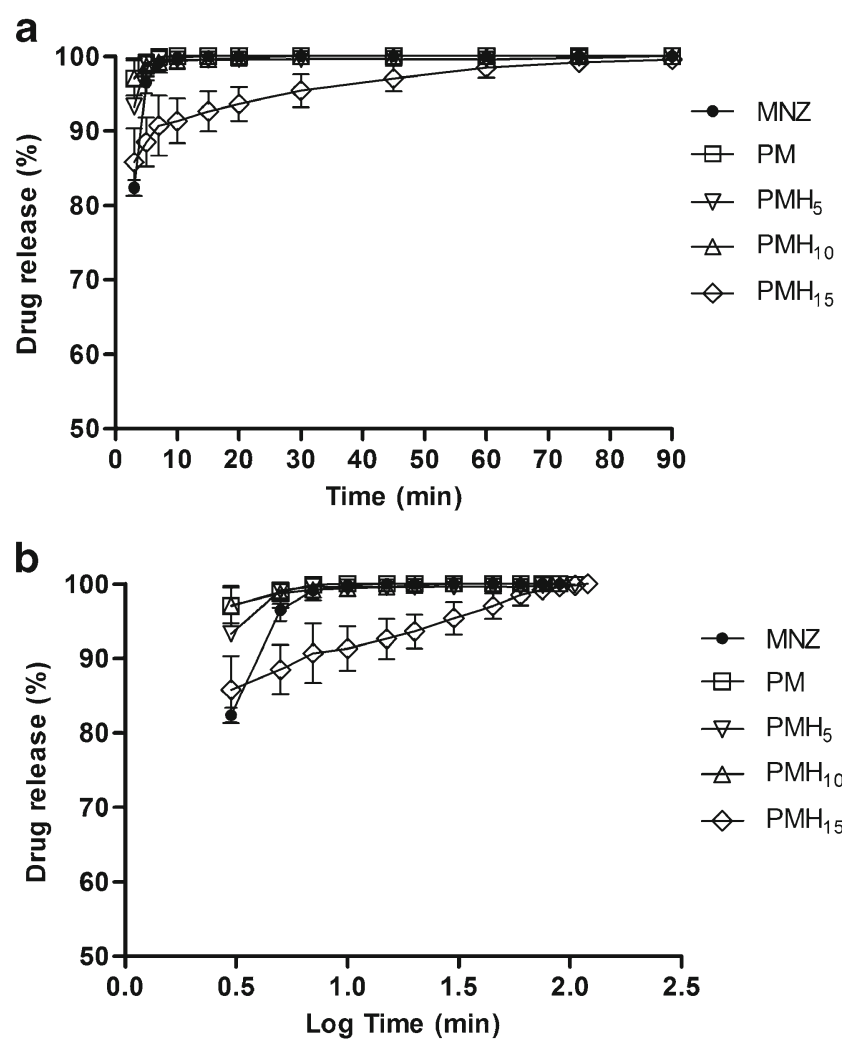

Fig. 8. Dissolution profiles of various microparticles at $\mathrm{pH}$ 7.4: $\mathbf{a}$ as a function of time and $\mathbf{b}$ as a function of log time

equivalent amount of MNZ but without HPMC. Compared to the corresponding physical mixture, the percentage reduction in MNZ crystallinity in microparticles of $\mathrm{PM}, \mathrm{PMH}_{5}, \mathrm{PMH}_{10}$ and $\mathrm{PMH}_{15}$ was $75.3,85.0,83.5$ and $78.6 \%$, respectively. Collectively, the results showed that the spray congealing process reduced the MNZ crystallinity to a much greater extent than HPMC. The reduction in MNZ crystallinity by HPMC was dependent on the concentration of HPMC. Several other studies have shown the ability of HPMC to decrease the crystallinity of the drug such as albendazole, felodipine, nicotinamide, acetaminophen, carbamazepine and trans-resveratrol by incorporating into the drug crystal lattice, having molecular interaction with the drug molecules or adsorbing onto the surface of the drug, thereby preventing crystal growth (9-11,31-33). However, the addition of HPMC into the formulation does not necessarily lead to a reduction in drug crystallinity as seen in other studies on omeprazole and prednisolone $(34,35)$.
The XRD data were further supported by the results of the DSC studies. Small MNZ endothermic peaks were observed for the physical mixtures $\mathrm{PMH}_{10}$ * and $\mathrm{PMH}_{15}$, with enthalpy of 4.46 and $4.60 \mathrm{~J} / \mathrm{g}$, respectively. The higher concentration of HPMC in the formulation prevented complete dissolution of the drug in PEG. The corresponding $\mathrm{PMH}_{10}$ and $\mathrm{PMH}_{15}$ microparticles also showed very small MNZ endothermic peaks, but their enthalpy of 3.21 and $3.64 \mathrm{~J} / \mathrm{g}$, respectively, were significantly lower $(p<0.05)$. This significant reduction in enthalpy of fusion in the microparticles compared to the corresponding physical mixture indicated decrease in MNZ crystallinity $(36,37)$.

The drug can be present as small crystalline particulates, amorphous particulates or it may be molecularly dispersed within the PEG matrix $(38,39)$. Therefore, Raman spectroscopy was used to further elucidate the solid state structure of the drug and the homogeneity of the drug distribution in the microparticles. The characteristic peak of the $\mathrm{NO}_{2}(\mathrm{~N}-\mathrm{O}$ stretching) of MNZ at $1528 \mathrm{~cm}^{-1}$ was observed in the spectra of microparticles with MNZ, indicating the presence of MNZ on the microparticle surface. This suggests that the drug was not totally embedded in the core of the microparticles when spray-congealed and that the concentration of HPMC up to $15 \%$ was not sufficient to fully coat the microparticle surface. Materials of crystalline structure are characterised by distinct and well-defined peaks in the Raman spectrum. The presence of drug peaks in the XRD and Raman spectra and endothermic peak in DSC curves indicate the presence of crystalline MNZ in the microparticles. If the percentage of the drug is high, it will form small crystals within the microparticles (40). In light of this, solubility of MNZ in PEG was determined and found to be $10.67 \%(w / w)$ at $80^{\circ} \mathrm{C}$. The theoretical percentage weight of the drug with respect to PEG in PM $(11.11 \%)$, $\mathrm{PMH}_{5}(11.76 \%), \mathrm{PMH}_{10}(12.50 \%)$ and $\mathrm{PMH}_{15}$ (13.33\%) was higher than the solubility limit. This aptly explains the presence of small crystals in those microparticles, accounting for peaks in the Raman spectra. Taking into account that there was a decrease in drug crystallinity, the remaining portion of MNZ within the microparticles could exist in the amorphous form and/or dispersed molecularly in PEG.

Drug-matrix interactions, such as hydrogen bonding, can be used to demonstrate the existence of drug as a molecular dispersion in the matrix (39). Both Raman spectroscopy and FT-IR spectroscopy were used to investigate the possible interactions of MNZ with PEG or HPMC. Collectively, the MNZ-PEG interaction observed in PM indicates the existence of MNZ as a molecular dispersion in the PEG matrix. Formation of hydrogen bonds between HPMC and MNZ further hindered recrystallisation of the molecularly dispersed drug

Table IV. Reasons for Chosen Additives

\begin{tabular}{ll}
\hline Additives & Reasons \\
\hline Dicalcium phosphate $(\mathrm{DCP})$ & Insoluble in water; reduces the hydration of microparticles \\
Magnesium stearate $(\mathrm{MgSt})$ & Hydrophobic excipient; relatively more hydrophobic than DCP \\
Methylcellulose $(\mathrm{MC})$ & Hydrophilic excipient; ability to swell and gel together with HPMC \\
Polyvinylpyrrolidone $(\mathrm{PVP})$ & Hydrophilic excipient; ability to swell, bind with the PEG matrix and gel together with HPMC \\
Silicon dioxide $\left(\mathrm{SiO}_{2}\right)$ & Hydrophilic excipient; Increases speed of hydration for faster swelling of HPMC and barrier formation \\
Sodium oleate/Citric acid & Formation of a coat by sodium oleate in an acidic environment \\
\hline
\end{tabular}



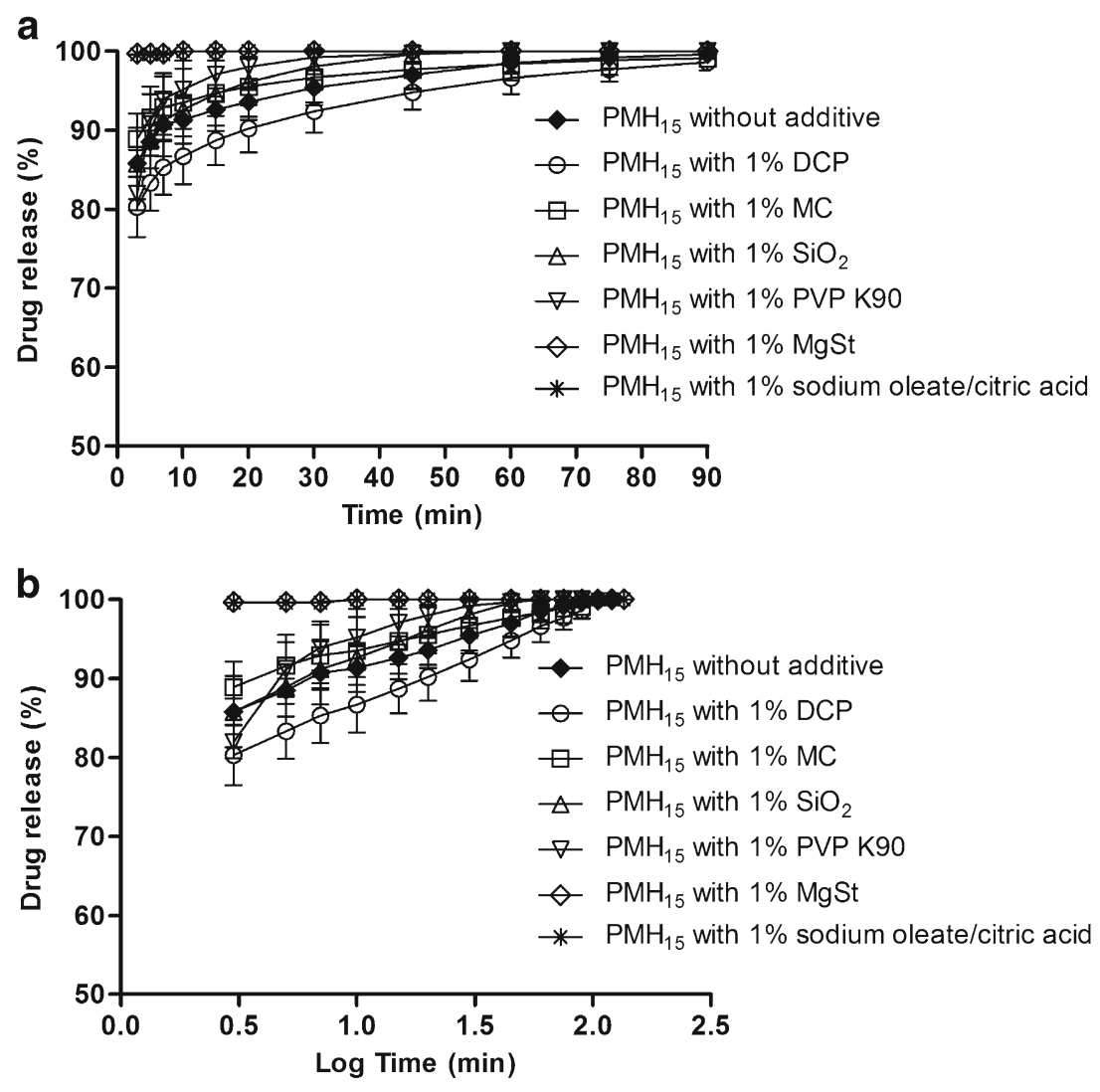

Fig. 9. Dissolution profiles of spray-congealed microparticles with various additives at $1 \%$ concentration: $\mathbf{a}$ as a function of time and $\mathbf{b}$ as a function of log time
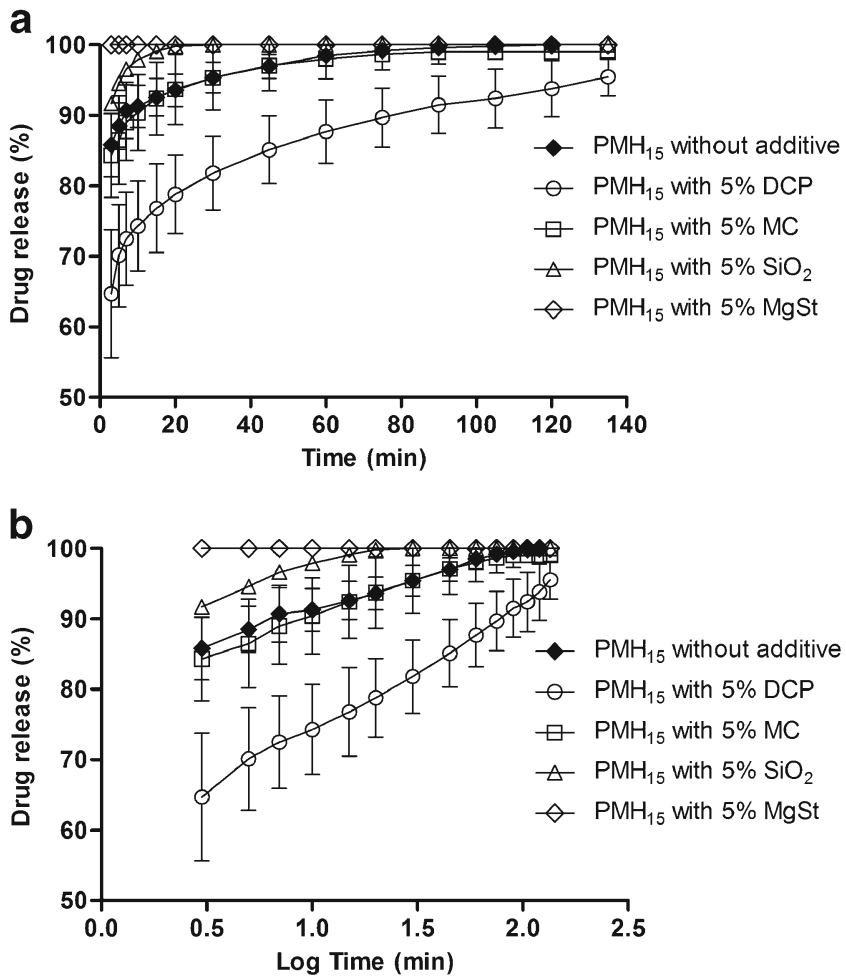

Fig. 10. Dissolution profiles of spray-congealed microparticles with various additives at $5 \%$ concentration: $\mathbf{a}$ as a function of time and $\mathbf{b}$ as a function of log time. Note: Formulation with 5\% PVP K90 was too viscous to spray as indicated by the reduction in MNZ crystallinity in the earlier XRD and DSC studies (Figs. 2 and 5).

\section{Impact of HPMC Particle Size on MNZ Crystallinity}

Mean percentage reduction in MNZ crystallinity of freshly spray-congealed (week 0) microparticles without HPMC (control) was approximately $75 \%$. This demonstrates that the spray congealing process was able to alter the solid state of the drug, leading to a reduction in drug crystallinity. In addition to MNZ-PEG interaction, the rapid cooling and solidification of the molten droplets also hindered the drug molecules from rearrangement into its crystalline form. Spraycongealed microparticles were also stable over 3 months (week 12) when stored at $25^{\circ} \mathrm{C}$ and $30 \% \mathrm{RH}$ as the percentage reduction in drug crystallinity at different time intervals did not change significantly. Metronidazole largely remained in the non-crystalline form after spray congealing.

Generally, all formulations containing HPMC exhibited a greater reduction in drug crystallinity. As mentioned previously, the MNZ-PEG interaction enabled the drug to exist as a molecular dispersion, reducing MNZ crystallinity of the spraycongealed microparticles. The drug could also exist as amorphous particles. HPMC particles could serve as a nucleating site, allowing MNZ molecules to deposit on the surface of the particle and orientate in a random and haphazard manner, leading to formation of amorphous particles. Formulations A5 and $\mathrm{A}-10 \%$ showed the greatest reduction in drug crystallinity (82.9 and $81.9 \%$, respectively) compared to all other 
formulations. It could be postulated that smaller HPMC particles $(33 \mu \mathrm{m})$ delayed nucleation and crystal growth of MNZ more than larger particles. At the same concentration, HPMC with a smaller particle size would be present in a larger number leading to a higher probability of the MNZ molecules adsorbing onto the surface of the particles. Hence, fewer crystals were formed, resulting in lower drug crystallinity. Larger HPMC particles of $59 \mu \mathrm{m}$ and above decreased the crystallinity of MNZ to a similar extent. Therefore, there was a critical HPMC particle size, beyond which changes in HPMC particle size had little effect on MNZ crystallinity.

No significant differences in the percentage reduction in drug crystallinity were observed between 5 and 10\% HPMC concentration of the same mesh size, leading to the inference that particle size played a greater role in reduction of drug crystallinity than the HPMC concentration studied.

\section{Modification of MNZ Release Using Various Additives}

Even though MNZ is poorly water soluble, the particles dissolved rapidly due to the small particle size of the drug and the sink condition of the dissolution medium. Therefore, marked increase in dissolution rate of MNZ from the spraycongealed microparticles would not be expected.

It was clearly seen that the $\mathrm{PM}, \mathrm{PMH}_{5}$ and $\mathrm{PMH}_{10}$ microparticles showed comparable dissolution rates which were slightly greater than that of the pure MNZ. This shows that spray congealing is able to enhance the dissolution of MNZ through reduction in MNZ crystallinity to a certain extent. HPMC is commonly used in preparations to modify drug release. Addition of 5 or $10 \%$ w/w $\mathrm{HPMC}\left(\mathrm{PMH}_{5}\right.$ and $\mathrm{PMH}_{10}$ ) further reduced the MNZ crystallinity by 3 to $10 \%$, but this did not further enhance the release of MNZ.

Burst effect of drug was observed for all microparticles, indicating that drug particles were present on the outer layer of the microparticle, which dissolved rapidly upon contact with the dissolution medium. The presence of MNZ on the surface of the microparticles was previously indicated by Raman spectroscopy. The results showed that the barrier to drug release did not form spontaneously upon hydration. The HPMC particles required time to hydrate and swell, to form the barrier. Interestingly, $\mathrm{PMH}_{15}$ was found to have a slower dissolution than $\mathrm{PM}, \mathrm{PMH}_{5}$ and $\mathrm{PMH}_{10}$. It was possible that the concentration of HPMC $(15 \% w / w)$ in $\mathrm{PMH}_{15}$ was sufficient to form a layer of gel that acted as a barrier to drug release. Upon hydration, the individual HPMC particles swelled to form clusters. These clusters would eventually merge, forming a barrier surrounding the microparticle, thereby modifying drug release.

When low additive concentration was used, formulations with sodium oleate/citric acid or $\mathrm{MgSt}$ had the fastest drug release. The coat layer formed by sodium oleate/citric and the hydrophobic MgSt could have retarded the wetting of HPMC particles by water. Without the wetting and swelling of HPMC particles, no barrier could be formed. Formulations containing PVP K90, MC or $\mathrm{SiO}_{2}$ all showed faster drug release than $\mathrm{PMH}_{15}$ while the formulation with DCP showed the opposite. Being hydrophilic, PVP K90 attracted water into the PEG matrix and the amount present was probably insufficient to exact the desired binding and gelling effect. Similarly, the hydrophilic $\mathrm{SiO}_{2}$ and MC attracted water in the PEG matrix, causing it to disintegrate before the HPMC particles could hydrate to form a barrier. On the other hand, the insoluble DCP reduced the hydration of the PEG matrix to an appropriate degree to prevent its disintegration, which allowed the HPMC particles to have sufficient time to hydrate, swell and form the barrier.

At $5 \%, \mathrm{MgSt}$ resulted in faster drug release than $1 \%$. This could be attributed to the greater hydrophobic effect of a higher concentration of MgSt. Thus, the hydrophobicity of an additive has to be carefully considered as it would affect the hydration of HPMC and consequently the drug release rate. At 5\%, the hydrophilic $\mathrm{SiO}_{2}$ attracted more water into the PEG matrix than $1 \%$, causing the rapid disintegration of the PEG matrix and resulted in even faster drug release. On the other hand, $\mathrm{MC}$ produced a greater gelling effect at higher concentration, leading to slower drug release than $1 \% \mathrm{MC}$. More interestingly, the addition of DCP at 5\% markedly reduced the release of drug, with $87.7 \% \mathrm{MNZ}$ released at $60 \mathrm{~min}$. The hydration and disintegration of the PEG matrix was markedly retarded by the higher concentration of DCP, allowing more HPMC to swell and form a more effective barrier.

From the above results, hydrophilic non-gelling additives could enhance the hydration rate of the microparticles, but they were ineffective for decreasing the gellation time of HPMC to form a barrier because of the rapid disintegration of the PEG matrix. The latter could be achieved by employing a high concentration of DCP. DCP reduced the hydration rate and retarded the disintegration of the PEG matrix, thus allowing sufficient time for HPMC to swell and form a barrier.

\section{CONCLUSION}

HPMC was successfully incorporated into spraycongealed PEG-MNZ microparticles where it exerted a significant effect on the crystallinity and release of MNZ. The MNZ release was more affected by the ability of HPMC to form a diffusion barrier than its ability to modifying MNZ crystallinity. Generally, spherical and free flowing spraycongealed microparticles with good drug encapsulation efficiency were obtained. Spray congealing was found to decrease MNZ crystallinity, and the incorporation of HPMC reduced the crystallinity further. The MNZ in the microparticles existed as crystalline particles, amorphous particles, as well as a molecular dispersion. Particle size of HPMC played an important role, with smaller HPMC particles $(33 \mu \mathrm{m})$ producing greater percentage reduction in MNZ crystallinity. Spraycongealed microparticles did not show any significant change in drug crystallinity over 3 months of storage at $25^{\circ} \mathrm{C}$ and $30 \%$ RH. Modified drug release of spray-congealed PEG-HPMC microparticles was successfully achieved with the addition of $5 \%$ DCP. Other additives added, such as MgSt, MC, PVP, $\mathrm{SiO}_{2}$ and sodium oleate/citric acid did not sufficiently modify the drug release from the microparticles.

\section{ACKNOWLEDGMENTS}

They would like to acknowledge the financial support from GEA-NUS Pharmaceutical Processing Research Laboratory fund (N-148-000-008-001).

Conflict of Interest The authors declare no conflict of interest. 


\section{REFERENCES}

1. Zhang GGZ, Zhou D. Crystalline and amorphous solids. In: Yihong Q, Yisheng C, Geoff GZZ, Lirong L, William RP, editors. Developing solid oral dosage forms. San Diego: Academic; 2009. p. 25-60.

2. Haleblian JK. Characterization of habits and crystalline modification of solids and their pharmaceutical applications. J Pharm Sci. 1975;64(8):1269-88.

3. Byrn S, Pfeiffer LR, Ganey LM, Hoiberg C, Poochikian G. Pharmaceutical solids: a strategic approach to regulatory considerations. Pharm Res. 1995;12(7):945-54.

4. Fahr A, Liu X. Drug delivery strategies for poorly water-soluble drugs. Expert Opin Drug Deliv. 2007;4(4):403-16.

5. Janssens S, Van den Mooter G. Review: physical chemistry of solid dispersions. J Pharm Pharmacol. 2009;61(12):1571-86. doi:10.1211/jpp.61.12.0001.

6. Raghavan SL, Schuessel K, Davis A, Hadgraft J. Formation and stabilisation of triclosan colloidal suspensions using supersaturated systems. Int J Pharm. 2003;261(1-2):153-8. doi:10.1016/S03785173(03)00299-0.

7. Simonelli AP, Mehta SC, Higuchi WI. Inhibition of sulfathiazole crystal growth by polyvinylpyrrolidone. J Pharm Sci. 1970;59(5):633-8.

8. Terayama H, Inada K, Nakayama H, Yasueda S, Esumi K. Preparation of stable aqueous suspension of a hydrophobic drug with polymers. Colloids Surf B: Biointerfaces. 2004;39(4):159-64.

9. Katzhendler I, Azoury R, Friedman M. Crystalline properties of carbamazepine in sustained release hydrophilic matrix tablets based on hydroxypropyl methylcellulose. J Control Release. 1998;54:69-85.

10. Kohri N, Yamayoshi Y, Xin HE, Iseki KEN, Sato N, Todo S, et al. Improving the oral bioavailability of Albendazole in rabbits by the solid dispersion technique. J Pharm Pharmacol. 1999;51(2):159-64. doi:10.1211/0022357991772277.

11. Konno H, Taylor LS. Influence of different polymers on the crystallization tendency of molecularly dispersed amorphous felodipine. J Pharm Sci. 2006;95(12):2692-705. doi:10.1002/jps.20697.

12. Rodriguez L, Albertini B, Passerini N, Cavallari C, Giovannelli L. Hot air coating technique as a novel method to produce microparticles. Drug Dev Ind Pharm. 2004;30:913-23.

13. Witschi C, Doelker E. Residual solvents in pharmaceutical products: acceptable limits, influence on physicochemical properties, analytical methods, and documented values. Eur J Pharm Biopharm. 1997;43:215-42.

14. Savolainen M, Khoo C, Glad H, Dahlqvist C, Juppo AM. Evaluation of controlled-release polar lipid microparticles. Int $\mathrm{J}$ Pharm. 2002;244(1-2):151-61.

15. Passerini N, Albertini B, Perissutti B, Rodriguez L. Evaluation of melt granulation and ultrasonic spray congealing as techniques to enhance the dissolution of praziquantel. Int J Pharm. 2006;318(1-2):92-102.

16. Ilic I, Dreu R, Burjak M, Homar M, Kerč J, Srčič S. Microparticle size control and glimepiride microencapsulation using spray congealing technology. Int J Pharm. 2009;381(2):176-83.

17. Mansur HS, Oréfice RL, Mansur AAP. Characterization of poly(vinyl alcohol)/poly(ethylene glycol) hydrogels and PVA-derived hybrids by small-angle X-ray scattering and FTIR spectroscopy. Polymer. 2004;45(21):7193-202.

18. Derosa RL, Trapasso JA. Poly(ethylene glycol) interactions with alumina and silica powders determined via DRIFT. J Mater Sci. 2002;37(6):1079-82.

19. Herculano RD, Alencar de Queiroz AA, Kinoshita A, Oliveira Jr ON, Graeff CFO. On the release of metronidazole from natural rubber latex membranes. Mater Sci Eng C. 2011;31(2):272-5. doi:10.1016/j.msec.2010.09.007.

20. Zaccaron CM, Oliveira RVB, Guiotoku M, Pires ATN, Soldi V. Blends of hydroxypropyl methylcellulose and poly(1-vinylpyrrolidone-co-vinyl acetate): miscibility and thermal stability. Polym Degrad Stab. 2005;90(1):21-7. doi:10.1016/j.polymdegradstab.2005.02.010.

21. Dhirendra K, Lewis S, Udupa N, Atin K. Solid dispersions: a review. Pak J Pharm Sci. 2009;22(2):234-46.

22. Fini A, Cavallari C, Ceschel G, Rabasco AM. Bimodal release of olanzapine from lipid microspheres. J Pharm Sci. 2010;99:4251-60.

23. Scalia S, Traini D, Young PM, Di Sabatino M, Passerini N, Albertini B. Comparison of spray congealing and melt emulsification methods for the incorporation of the water-soluble salbutamol sulphate in lipid microparticles. Pharm Dev Technol. 2013;18:266-73.

24. Savolainen M, Herder J, Khoo C, Lovqvist K, Dahlqvist C, Glad $\mathrm{H}$, et al. Evaluation of polar lipid-hydrophilic polymer microparticles. Int J Pharm. 2003;262:47-62.

25. Albertini B, Mezzena M, Passerini N, Rodriguez L, Scalia S. Evaluation of spray congealing as technique for the preparation of highly loaded solid lipid microparticles containing the sunscreen agent, avobenzone. J Pharm Sci. 2009;98:2759-69.

26. Passerini N, Perissutti B, Moneghini M, Voinovich D, Albertini B, Cavallari C, et al. Characterization of carbamazepine-Gelucire $50 / 13$ microparticles prepared by a spray-congealing process using ultrasounds. J Pharm Sci. 2002;91:699-707.

27. Passerini N, Perissutti B, Albertini B, Voinovich D, Moneghini M, Rodriguez L. Controlled release of verapamil hydrochloride from waxy microparticles prepared by spray congealing. J Control Release. 2003;88:263-75.

28. Albertini B, Passerini N, González-Rodríguez ML, Perissutti B, Rodriguez L. Effect of Aerosil $®$ on the properties of lipid controlled release microparticles. J Control Release. 2004;100:233-46.

29. Collett JH, Flood BL, Sale FR. Some factors influencing dissolution from salicylic acid urea solid dispersions. J Pharm Pharmacol. 1976;28:305-8.

30. Sjokvist Saers E, Nystrom C, Alden M. Physicochemical aspects of drug release. XVI. The effect of storage on drug dissolution from solid dispersions and the influence of cooling rate and incorporation of surfactant. Int J Pharm. 1993;90:105-18.

31. Hino T, Ford JL. Characterization of the hydroxypropylmethylcellulosenicotinamide binary system. Int J Pharm. 2001;219:39-49.

32. Kim S, Ng WK, Dong Y, Das S, Tan RBH. Preparation and physicochemical characterization of trans-resveratrol nanoparticles by temperature-controlled antisolvent precipitation. J Food Eng. 2012;108:37-42.

33. Wen H, Morris KR, Park K. Synergic effects of polymeric additives on dissolution and crystallization of acetaminophen. Pharm Res. 2008;25:349-58.

34. Wolf B. Bead cellulose products with film formers and solubilizers for controlled drug release. Int J Pharm. 1997;156:97-107.

35. Sarisuta N, Kumpugdee M. Crystallinity of omeprazole in various film polymers. Pharm Pharmacol Commun. 2000;6:7-11.

36. Elkordy AA, Essa EA. Dissolution of ibuprofen from spray dried and spray chilled particles. Pak J Pharm Sci. 2010;23(3):284-90.

37. Gill P, Moghadam TT, Ranjbar B. Differential scanning calorimetry techniques: applications in biology and nanoscience. $\mathrm{J}$ Biomol Tech. 2010;21(4):167-93.

38. Craig DQM. The mechanisms of drug release from solid dispersions in water-soluble polymers. Int J Pharm. 2002;231(2):131-44. doi:10.1016/s0378-5173(01)00891-2.

39. Chiou WL, Riegelman S. Pharmaceutical applications of solid dispersion systems. J Pharm Sci. 1971;60(9):1281-302.

40. Leuner C, Dressman J. Improving drug solubility for oral delivery using solid dispersions. Eur J Pharm Biopharm. 2000;50:47-60. 